\title{
Research on the Control of Fuel Regulator Overshoot Based on Linear Ultrasonic Motor
}

\author{
Tian Gan* , Li Yin , Yan Huipeng , Yang Zhengwei , Zhang Wei \\ Xi'an Research Inst. Of Hi-Tech Hongqing, Xi'an.P.R.China,710025
}

(Tel: 86-13152187219, E-mail: tiangan_2012@163.com)

\begin{abstract}
Keywords: Linear ultrasonic motor, Fuel regulator, Overshoot, Successive approximation, Speed regulation

Abstract. The fuel regulator based on the linear ultrasonic motor can effectively control the fuel flow. However, the slip of the actuator in the braking phase will cause the overshoot, so that the fuel regulator can not control the fuel flow accurately. In order to reduce the overshoot, this paper presents the result control method based on the successive approximation and the process control method based on the speed regulation by analyzing the representative form of the overshoot of the regulator and the main cause of the overshoot. The control program is designed and programmed. The experimental results show that the two control methods can greatly reduce the overshoot of the fuel regulator, but the adjustment time has increased. Over comprehensive measure of overshoot and adjustment time, the result control based on successive approximation is better than process control based on speed regulation. The overshoot of the fuel regulator based on linear ultrasonic motor is reduced about $70 \%$ compared to that of traditional fuel regulator when the result control based on successive approximation is adopted, which greatly improves the fuel flow control accuracy.
\end{abstract}

\section{Introduction}

During the flight, the fuel flow control system for aircraft engine constantly changes the supply of fuel according to the status of the engine, in order to control the engine thrust output, so as to adjust the flight condition, the key components of the fuel supply regulator is fuel flow regulator, and its performance is good or bad directly affect the engine performance[1-3]. But the current supply of turbofan engine fuel control regulator has a lot of problems, such as, low speed, poor electromagnetic compatibility and low regulation accuracy. Therefore, it is necessary to study a new fuel flow control mode. Linear ultrasonic motor, as a new type motor that can directly drive the load, with high control precision, no electromagnetic interference and simple structure, has broad application prospects in the field of micro precision control, and provides a new way for the realization of the turbofan engine fuel flow control[4-7]. An important indicator to measure the performance of the fuel supply regulator is the accuracy, and the overshoot is the key parameters reflecting the fuel regulating accuracy. Overshoot will cause the fuel supply regulator not accurately control the fuel flow, thus reducing the overshoot is of great significance for the realization of accuracy of the fuel flow regulator[8-10].

Therefore, based on the analysis of the linear ultrasonic motor working principle, through the analysis of overshoot representation and the main causes, this paper puts forward two kinds of overshoot control methods. The combination of programming design and experimental study is for comparative analysis, so as to obtain the optimal control method and provide effective means to improve the control precision of the fuel flow.

\section{The structure and working principle of linear ultrasonic motor}

The structure of linear ultrasonic motor is shown in Fig. 1, which mainly comprises a stator and a rotor. The stator comprises a rear cover, a front cover, a support plate and a piezoelectric ceramic plate, of which the function is to achieve the ultrasonic vibration of the stator based on the inverse piezoelectric effect of piezoelectric ceramics. The rotor includes slide and friction plate, of which the role is to output linear motion by converting the friction and drive load. The working principle of linear 
ultrasonic motor is: four pairs of piezoelectric ceramic pieces after polarization along the thickness direction (each piezoelectric ceramic plate in the opposite direction of polarization), under the excitation of alternating voltage, generates micro longitudinal vibration owing to the inverse piezoelectric effect of itself, thus boosting the stator vibration. The vibration by the friction effect of the rotor friction layer, is converted into the linear motion of the slider, so as to drive the load.

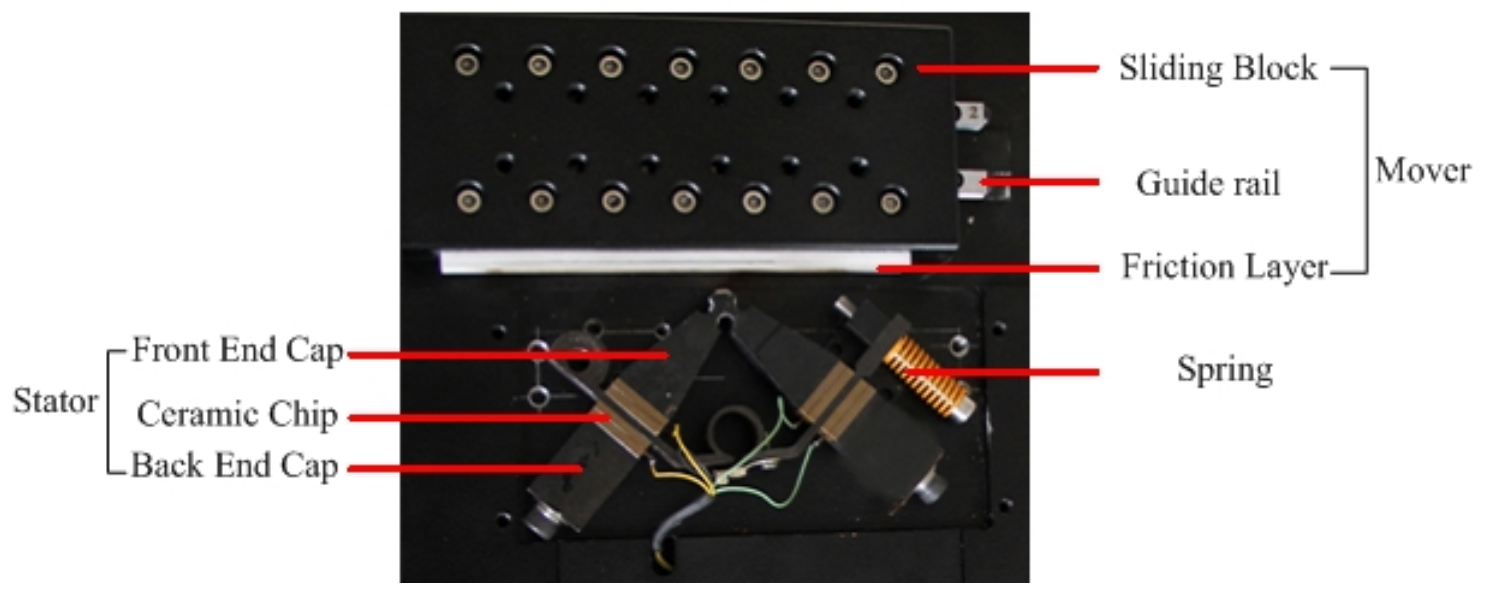

Fig. 1 Physical Diagram of Linear Ultrasonic Motor

The working process of linear ultrasonic motor in one cycle is as shown in Fig. 2. 1, 3 denote the antisymmetric mode; 2, 4 denotes the symmetric mode; Ellipse represents the motion trajectory of the stator driven surface particle; Arrow denotes the movement direction of the rotor. Apply the same sine signal $(\sin w t)$ for the two pairs of the piezoelectric ceramic piece, which is in the half bottom in the Fig.1, then the vibrator is stretched or contracted at the same time, exciting the symmetric mode of the stator; Apply opposite cosine signal (coswt and -coswt) for the top two pairs of piezoelectric ceramic pieces, then the vibrator is stretched or contracted at the same time in opposite direction, exciting the antisymmetric mode of the stator. Therefore, if apply the signal metioned above to 4 pairs of piezoelectric ceramic pieces at the same time, it can both stimulate symmetric and antisymmetric mode of the stator. Figure 2 shows that the working process of the motor is $1-2-3-4-1$, and the working process can also be carried out in accordance with the order of 1-4-3-2-1,just needing adjusting the phase difference of excitation signal.

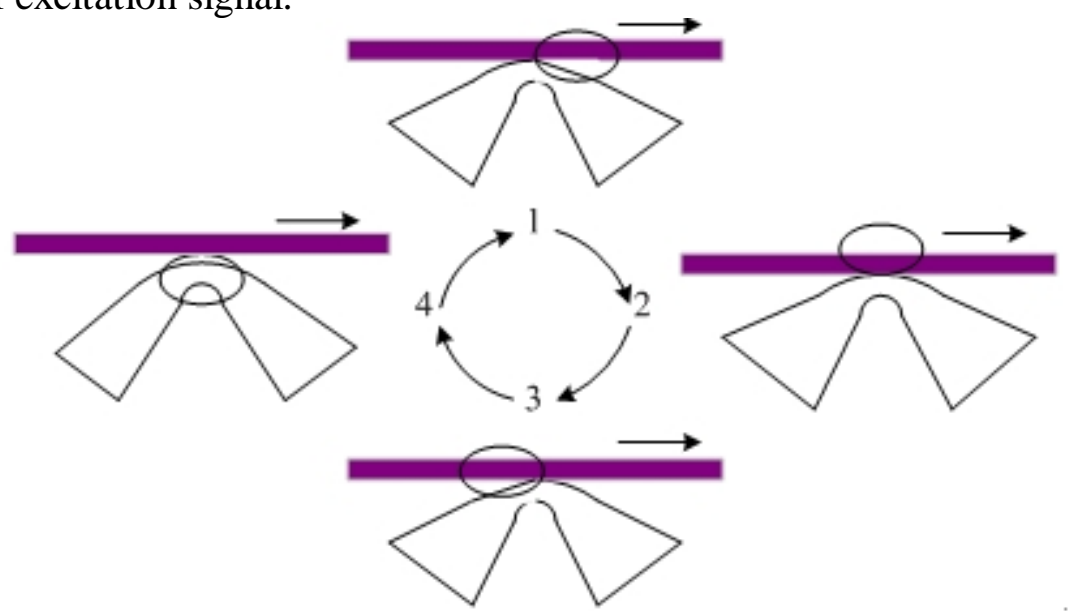

Fig. 2 Schematic diagram of linear ultrasonic motor

\section{Overshoot experiment of fuel regulator based on linear ultrasonic motor}

The experiments are based on the linear ultrasonic motor-based fuel regulator experimental platform, as shown in Fig. 3. The motion process of fuel regulator actuator includes acceleration, uniform speed and deceleration. The displacement of acceleration process is $6 \mathrm{~mm}$, and grating encoder accuracy is $4 \mu \mathrm{m}$. When the input pulse number is set to less than 1500 , the actuator is in the acceleration state. When the number of input pulses is set to more than 1500 , the actuator is in uniform 
state finally. In view of this, in order to comprehensively study the overshoot of fuel supply regulator in two stages of acceleration and uniform speed, the input pulse number must be set above 1500 .

Therefore, set the number of input pulses from 100 to 2000 and the interval is 100. In the experiment, the positioning mode is adopted, and the overshoot of the controller is obtained by comparing the input pulse number and the output pulse number. In order to ensure the accuracy of the experiment and reduce the experimental error, the experimental method of averaging is used. The experimental results are shown in Fig.4 and Fig.5. Among them, the overshoot calculation formula is:

$$
\text { overshoot }=\frac{\text { output pulse number }- \text { input pulse number }}{\text { input pulse number }} \times 100 \%=\frac{\text { overshoot } \text { pulse number }}{\text { input pulse number }} \times 100 \%
$$

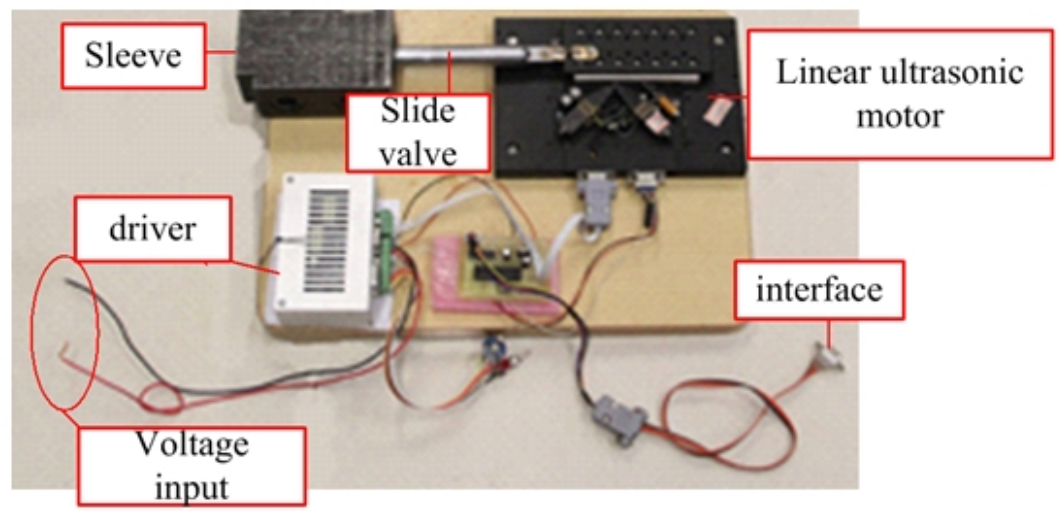

Fig. 3 Experimental Platform for Motion Mechanism of Actuators

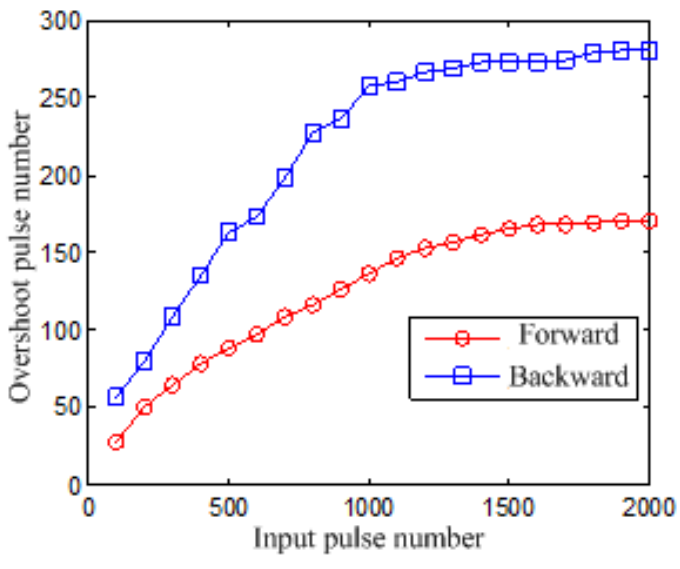

Fig. 4 Pulse overshoot curve

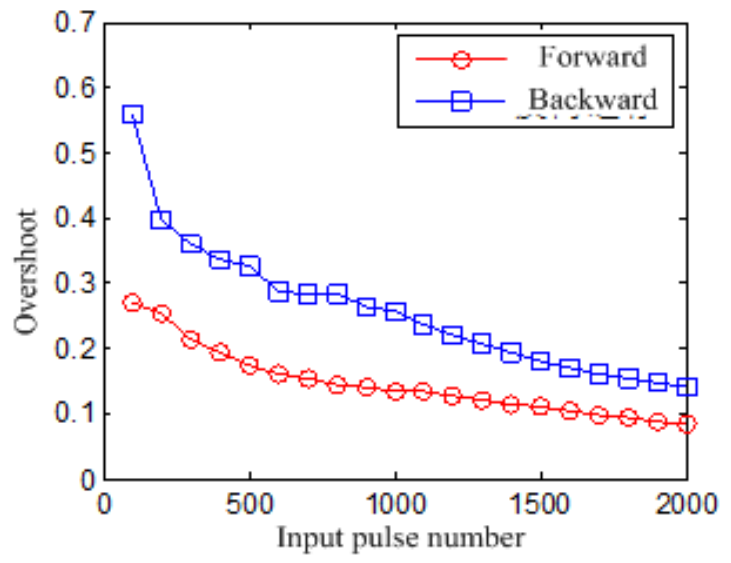

Fig. 5 Overshoot and input pulse curve

It can be seen from Fig.4 that the overshoot pulse number increases with the increasing number of input pulses in the acceleration phase; and the number of overshoot pulses is almost constant as the input pulse increases in the uniform speed phase. This is because in the acceleration phase, with the increase of the number of input pulses, the speed of actuator increases, and in the same braking state, slip distance is longer, so that overshoot pulse number is larger; in the uniform speed phase, the speed of actuator remains constant with the increasing number of input pulses, and in the same braking state, the slip distance is equal, so that the number of overshoot pulses is almost constant. Fig. 5 shows the relationship between overshoot and the number of input pulses. Although the number of overshoot pulses increases as the number of input pulses increases, the overshoot decreases gradually, and the decreasing trend gradually decreases. This is mainly due to the increasing denominator in the formula (2). At the same time, the above two figures also reflect that as the motor running direction is different, overshoot is also different. When the running direction is forward, the overshoot range is from $10 \%$ to $29 \%$; when the running direction is backward, the overshoot range is from $15 \%$ to $55 \%$. 


\section{Control method of overshoot}

The presence of overshoot causes that the fuel regulator fails to accurately control the fuel flow, so reducing the overshoot is important to achieve the accuracy of fuel flow regulation. In the above experiment, the representation form of overshoot is that the number of output pulses is larger than that of input pulses, so adjusting the number of input pulses by corresponding procedures can reduce overshoot. In addition, the main reason that overshoot generates is the slip of the actuator in the braking phase, so reducing the moving speed of the actuator, which can reduce the sliding distance in the braking phase, can reduce overshoot. In view of this, this paper proposes two overshoot control methods: the result control method based on successive approximation and process control method based on the speed regulation.

\section{Result control method based on successive approximation}

Set the input pulse number as $\mathrm{Y}$, and the output pulse number as $\mathrm{xi}, \mathrm{i}=(1,2,3 \ldots \mathrm{n})$. Adopt successive approximation method, and the output pulse number is calculated as:

$$
\left\{\begin{array}{l}
\text { error }=Y-x_{i} \\
x_{i+1}=x_{i}+w \cdot \text { error }
\end{array}\right.
$$

Where $w$ is the correction factor, error is the difference between the input pulse number and the output pulse number.

The basic idea of result control method based on successive approximation is: Before the control procedure is designed, the input pulse number, the initial output pulse number, the threshold value and the correction factor must be set first. When the fuel regulation starts, if the error is more than the threshold value, the target value can be quickly approached according to the formula (2); if the error is less than the threshold value, adopt the point control mode (ie, the actuator will move a few pulses at a time), so that the output pulse number gradually approaching the target value.

In this paper, the correction factor $\mathrm{w}=6 / 24$, the threshold is set to 10 , and the control process is shown in Fig. 6.

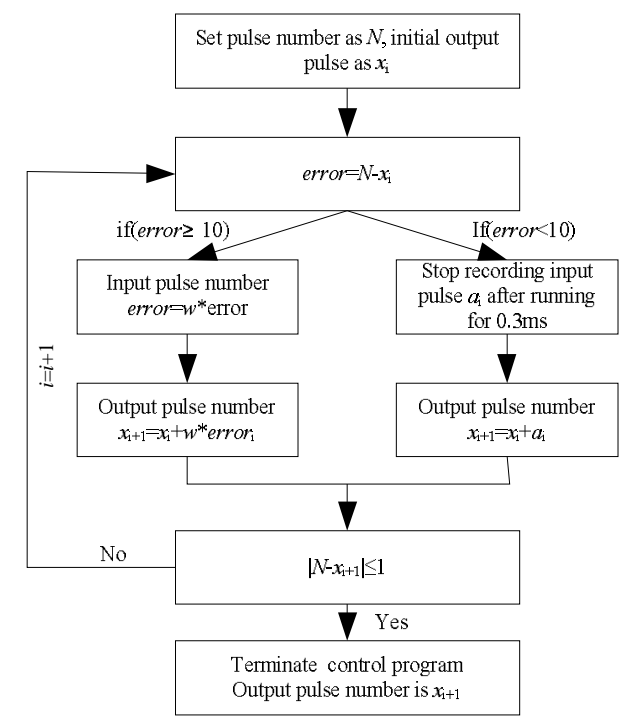

Fig 6 Results-based control flow based on successive approximation

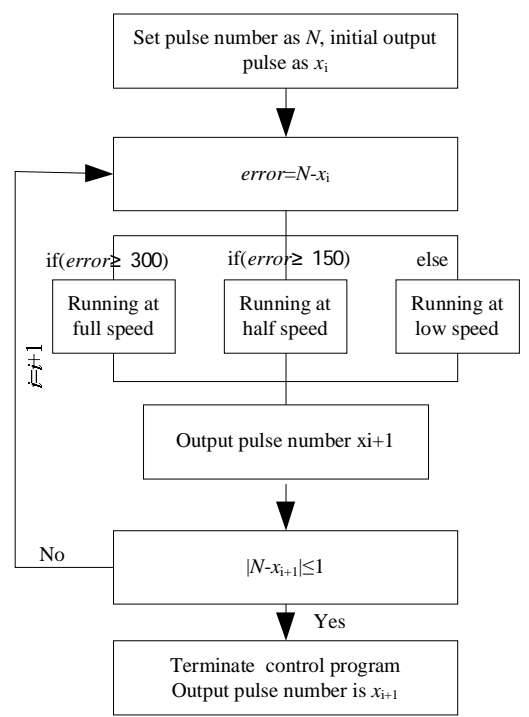

Fig. 7 Process control flow based on speed regulation

\section{Process control method based on speed regulation}

The basic idea of the process control method based on speed regulation is: Before the procedure is designed, the input pulse number, the initial output pulse number, threshold must be set firstly. When 
fuel regulation is in progress, initially due to the larger error, the actuator can run at a high speed, so that the number of output pulses can quickly close to the target value; as the error gradually decreases, the actuator speed can be reduced gradually, so that when the control is stopped, the running speed of the actuator is small and the slip distance is short. Therefore, the output pulse number can gradually reach the target value, thereby reducing the overshoot.

In the designed procedure, the threshold is set to 300, and the control process is shown in Fig. 7.

\section{Experimental Study}

From the previous study, the two main indicators measuring the performance of the fuel regulator is fuel regulation time and overshoot. In order to study the advantages and disadvantages of the two control methods, the result control procedure based on successive approximation and process control procedure based on speed regulation are both programmed into the control module in Fig. 3, and the other experimental parameters are the same with Section 2. By comparison of the fuel regulation time and the overshoot of the regulator under the two control methods, which control method is better can be judged. The overshoot calculation formula can be replaced by formula (1), and the fuel regulation time is calculated as follows:

$$
\text { fuel regulation time }=\frac{R x}{n \times T x} \times T s-T
$$

Where Rx is the byte number received by the station, Tx is the byte number sent by the station, Ts is the time interval between two adjacent output pulses and the value is $0.5 \mathrm{~ms}, \mathrm{n}$ is the number of experiments and the value is $10, \mathrm{~T}$ is the inherently extended sampling time set by the control module and the value is $20 \mathrm{~ms}$.

Respectively program the procedure of the two control method into the control module, and set the input pulse number from 100 to 2000 and the interval is 100. By observing and recording output pulse number that the station shows, the overshoot of the fuel regulator is studied. The results are as follows:

( a ) The comparative results between the two control methods under forward operation is as shown in Fig. 8 and Fig. 9.

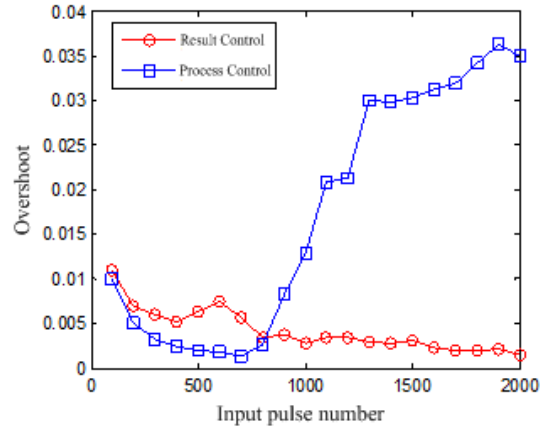

Fig. 8 The overshoot-input impulse relation under forward operation

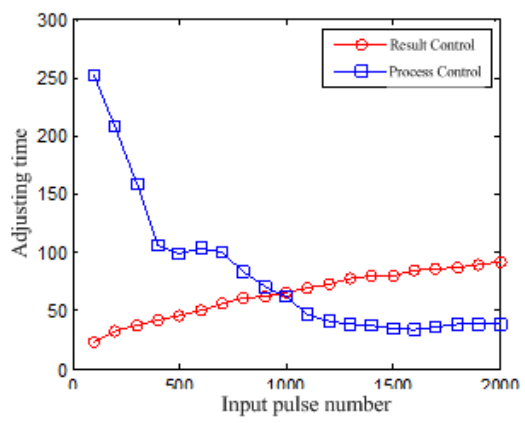

Fig. 9 Adjustment time -input pulse relationship under forward operation

( $b$ ) The comparative results between the two control methods under backward operation is as shown in Fig. 10 and Fig. 11. 


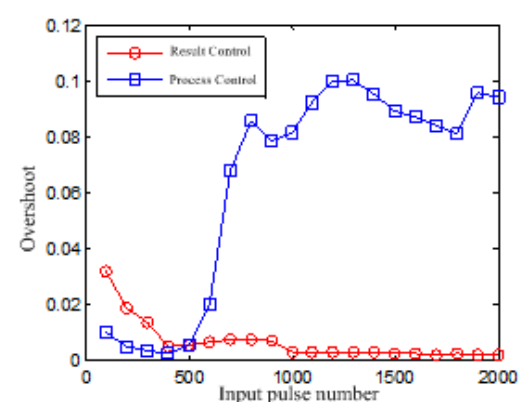

Fig. 10 The overshoot-input impulse relation under backward operation

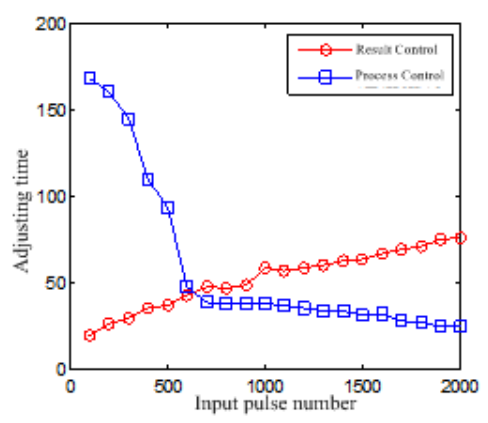

Fig. 11 Adjustment time -input pulse relationship under backward operation

From the above experimental results, it can be known that:

(1)When the actuator of the fuel supply regulator runs in the forward direction, the overshoot generated by the result control method based on successive approximation decreases with the increasing number of input pulses, and finally stabilizes. The maximum overshoot is $1 \%$. Fuel regulation time increases with the increasing number of the input pulse, and the maximum regulation time is $90 \mathrm{~ms}$; when it runs in the backward direction, the overshoot generated by the result control method based on successive approximation also decreases with the increasing number of input pulses, and finally stabilizes. The maximum overshoot is $3.5 \%$. The regulation time increases with the increasing number of the input pulse, and the maximum regulation time is $70 \mathrm{~ms}$.

(2)When the actuator of the fuel supply regulator runs in the forward direction, the overshoot generated by the process control method based on speed regulation decreases first and then increases with the increasing number of the input pulses. The maximum overshoot is $3.5 \%$. The regulation time increases with the increasing number of input pulses, and the maximum regulation time is $252 \mathrm{~ms}$; when it runs in the backward direction, the maximum overshoot is $10 \%$, and the maximum regulation time is $167 \mathrm{~ms}$.

(3)Whether the actuator of the fuel supply regulator is in the forward or backward direction, when the input pulse number is small (750 in the forward direction and 500 in the backward direction), the overshoot generated by the process control method based on speed regulation is slightly less than that generated by the result control method based on successive approximation; when the input pulse number is large, the overshoot generated by the process control method is much larger than that generated by the result control method. For the fuel regulation time, when the input pulse number is small (1000 in the forward direction, 600 in the backward direction), The fuel regulation time that the process control method requires is much greater than the result control method; When the input pulse number is large, the process control method is less than the result control method.

In order to ensure the high mobility of the vehicle, the ideal state of fuel flow regulation is fast and accuracy. Overshoot directly determines the fuel regulation accuracy, and fuel regulation time directly determines the fuel regulation fast. However, both the overshoot and the regulation time can not be balanced whether the actuator of the fuel supply regulator is in the forward or backward direction. When the overshoot is small, the regulation time is long and when the overshoot is large, the regulation time is short. It can be seen that from the above results that the two control methods proposed in this paper can greatly reduce the fuel overshoot, but the regulation time increases. The overshoot range generated by the result control method based on successive approximation is: $0.2 \% \sim 1 \%$ in the forward direction, $0.1 \% \sim 3.5 \%$ in the backward direction, and the regulation time range is: $20 \mathrm{~ms} \sim$ $90 \mathrm{~ms}$ in the forward direction, $\mathrm{r} 20 \mathrm{~ms} \sim 70 \mathrm{~ms}$ in the backward direction; The overshoot range generated by the process control method based on speed regulation is: $0.1 \% \sim 3.5 \%$ in the forward direction, $0.1 \% \sim 10 \%$ in the backward direction, and the regulation time range is: $250 \mathrm{~ms} \sim 40 \mathrm{~ms}$ in the forward direction, $165 \mathrm{~ms} \sim 25 \mathrm{~ms}$ in the backward direction. While the overshoot of the traditional turbofan engine fueling regulator is $9.79 \%$. 
From all the above, the result control method based on successive approximation is better than the process control method based on speed regulation. At the same time, when the result control method based on successive approximation is adopted, the overshoot generated by fuel supply regulator based on linear ultrasonic motor reduces by about $70 \%$ than the traditional fuel supply regulator, greatly improving the fuel control accuracy.

\section{Conclusion}

In order to realize precise control of the fuel flow of the turbofan engine, two kinds of control methods are proposed to reduce the overshoot. The control program is programmed and the overshoot of the fuel supply regulator is researched through the experiment. The main conclusions are as follows:

(1)The two control methods can greatly reduce the overshoot of the fuel supply regulator, but the fuel regulation time increases. Overshoot and fuel regulation time can not be balanced. When the overshoot is mall, the regulation time is long; when the overshoot is large, the regulation time is short.

(2)Based on the overshoot and fuel regulation time, the conclusion can be obtained that the result control method based on successive approximation is better than the process control method based on speed regulation through comprehensive comparison and analysis of the experimental results.

(3)When the result control method based on successive approximation is adopted, the overshoot generated by fuel supply regulator based on linear ultrasonic motor reduces by about $70 \%$ than the traditional fuel supply regulator, greatly improving the fuel control accuracy.

The above research shows that the fuel supply regulator based on linear ultrasonic motor can reduce overshoot of the fuel supply regulator and realize the precise control of the fuel flow by adopting the appropriate control method.

\section{References}

[1] Garraway G H, Kirkup R P. Fuel flow control[J]. 1956.

[2] Zhang S Y, Tao S J. Investigation of Digital Fuel Supply Regulator for Aeroengine[J]. Aeroengine, 2009.

[3] La F F, Sones W. Fuel supply system with pressure regulator[D]. US.

[4] Lin F J, Wai R J, Chen M P. Wavelet neural network control for linear ultrasonic motor drive via adaptive sliding-mode technique[J]. IEEE Transactions on Ultrasonics Ferroelectrics \& Frequency Control, 2003, 50(6):686-698.

[5] Zhang F, Chen W, Liu J, et al. Bidirectional linear ultrasonic motor using longitudinal vibrating transducers.[J]. IEEE Transactions on Ultrasonics Ferroelectrics \& Frequency Control, 2005, 52(1):134-138.

[6] Lu C, Xie T, Zhou T, et al. Study of a new type linear ultrasonic motor with double-driving feet.[J]. Ultrasonics, 2007, 44 Suppl 1(4):e585-9.

[7] Huang Guo-qing, LIU Qun-ting . Recent Advances in Linear Ultrasonic Motor[J] . Micromotor, 2003, (2): 24-26 .

[8] Wei ZHANG, Yin LI, Zhengwei YANG, etal . Analysis of Fuel Control System in Rocket Used by Linear Ultrasonic Motor [C] . 2014 International Academic Conference on Environment, Energy and Power Engineering, 2014: 807-812 .

[9] Seemann A A, Wolfgang . Ultrasonic traveling wave linear motor with improved effiency [C] . SPIE, Proceeding,1992, (2716): 554-564 .

[10] Yang Ming, Robert C. Richardson, Martin C. Levesley, et al . Perfomance Improvement of Rectangeular-Plate Linear Ultrasonic Motors Using Dual-Frequency Drive[J] . IEEE 
Transactions on Ultrasonic, Ferroelectrics, and Frequency Control, 2004, 51(12): 1600-1606. 